\section{El pescador en el imaginario científico durante la etapa de formación de la academia ictiológica chilena, 1829-1909*}

\section{Fishermen in the scientific imaginary during the formative stage of Chile's ichthyological academy, 1829-1909}

Jorge Muñoz Sougarret

Professor do Centro de Estudios del Desarrollo Local y Regional/Universidad de Los Lagos. Av. Cochrane, 1056 casilla 933

5310887 - Osorno - Provincia de Osorno - Chile munozsougarret@yahoo.es

\section{Francisco Ther Ríos}

Professor do Departamento de Ciencias Sociales/ Universidad de Los Lagos.

Av. Fuschlocher, 1305 casilla 933 5311157 - Osorno - Provincia de Osorno - Chile fther@ulagos.cl
MUÑOZ SOUGARRET, Jorge; THER RÍOS, Francisco. El pescador en el imaginario científico durante la etapa de formación de la academia ictiológica chilena, 1829-1909. História, Ciências, SaúdeManguinhos, Rio de Janeiro, v.20, n.4, out.-dez. 2013, p.1621-1633.

\section{Resumen}

A fines del siglo XIX, en Chile, se comenzaba a crear una academia científica. Amparada bajo el resguardo del Estado, compartió sus orientaciones y objetivos. El Estado solicitó a la ciencia que se abocara a aumentar la producción económica y social del país, modernizando las faenas tradicionales desde la cúspide. En nuestro trabajo nos centraremos en la relación entre ciencia y pesca, rescatando la visión del científico y su conflictivo diálogo con los pescadores artesanales. Infecunda relación que terminó con la petición científica de la deslegitimación del otro como productor, receptor y codificador del saber.

Palabras clave: ciencia; mundo científico; saberes tradicionales; Chile; siglo XIX.

\section{Abstract}

Chile began creating a scientific academy in the late nineteenth century. Coming under the umbrella of the state, it shared governmental perspectives and objectives. The state asked science to help augment the country's economic production and social development by modernizing traditional forms of work from the top down. In our article we focus on the relation between science and fishing, examining the vision of scientists and their conflict-ridden dealings with traditional fishermen. This was not a fruitful relationship, and it ended with scientists requesting the delegitimization of fishermen as producers, receivers and encoders of knowledge.

Keywords: science; scientific world; traditional knowledge; Chile; nineteenth century. 
$\mathrm{U}$ n primero de julio de 1999 se firmaba en la ciudad de Budapest la declaración sobre ciencia y saber científico (emanada de la conferencia mundial organizada por la Unesco y el Consejo Mundial de la Ciencia). Los signatarios, venidos desde los más disímiles rincones del globo, afirmaban ahí un nuevo patrón para entender la ciencia; eliminaban la odiosa supremacía de la razón e instauraban que las ciencias con vocación universalista debían respetar y aprender de todas las "creatividades humanas" (Unesco/ICSU, art.35, 1999). El júbilo que se instaló en los países en camino al desarrollo pareció contrastar la mudez de los consorcios privados y nacionales de los países productores de tecnología; habría de transcurrir menos de una década para que se hicieran oír voces que denunciaban la apropiación, por parte de las corporaciones ya enunciadas, del conocimiento de las sociedades tradicionales. Conocimiento que era transformado en una propiedad, desconociendo las implicancias históricas y culturales de su construcción (Tinnaluck, 2004; Gómez, Villar, 2009).

Amplia ha sido la historia del despojo de conocimiento, más limitado el estudio de los individuos que lo realizaron, sus impresiones y justificaciones. Nuestro estudio se centrará en ahondar en la situación particular dada en Chile, puntualmente en la relación entre los científicos y los pescadores artesanales. Las extensas costas del país, en comparación con su reducida continentalidad, presentan a Chile como un correcto laboratorio para examinar el diálogo creativo entre ciencia ictiológica y saber pesquero tradicional. Debido a las limitaciones editoriales, nos abocaremos a la primera etapa de la formación científica en el país (1829-1909), explorando las motivaciones políticas, ideológicas y sociales de los científicos y cómo la conjunción de los elementos enunciados condicionó su relación con los pescadores. Exponiendo enjutamente nuestra hipótesis al lector, los científicos se habrían negado a dialogar con los pescadores, exigiéndoles la entrega unilateral de información, en tanto abogaban públicamente por el desempoderamiento de tal grupo social.

\section{La red se cierne sobre los pescadores: la formación filosófica de la ciencia europea}

Al abrigo del Mediterráneo, a lo largo de varios miles de años, la intelectualidad occidental se vio compelida a dialogar con el mundo pesquero en búsqueda de alimentos y materiales de análisis (Braudel, 1979). Aquella simbiosis de barriga y mente comenzó a ser abandonada con la revolución cartesiana que personalizó al saber, considerando, en este giro anti aristotélico, a todos los elementos naturales como cognoscibles en su reducción abstracta como idea (Salles, 1993, p.293-296). La intelectualidad gala motivada por la nova agitación se propuso ahondar en la mente, desde las formas a las ideas, siendo Diderot y D'Alembert los gestores y la Encyclopédie su esfuerzo más ambicioso (Díaz de la Serna, 2009, p.156). Siguiendo a sus autores, es observable su certeza de que el hombre estaba guiado por la búsqueda del entendimiento, percibido como la visión completa y final de los elementos o situaciones, la verdad en su forma pura. El engaño de las formas debía ser superado por la experiencia, particularmente de tres tipos: memoria, razón e imaginación. Asociadas todas a tres ciencias: historia, filosofía y poesía.

Inserta en la primera experiencia hemos de encontrar a la pesca. Empero ser tangencial en relación al contenido central de la Encyclopédie, de la treintena de artículos relativos a ese oficio, un reducido número fue firmado, siendo siempre uno el firmante: Louis de Jaucourt 
(1704-1779), noble francés, consagrado a la medicina, que actuaría de presentador del mundo marino al lector. La última palabra dista de ser cándida. Jaucourt imaginó a la pesca como una herramienta, un intersticio entre el pez y su pescador; en tanto, las bellas artes y manufacturas eran exaltadas como el correcto hacer, la pesca fue incluida bajo las tradiciones; era un hacer por costumbre, exiliado de la imaginación y la historia. ${ }^{1}$

Objetos imperfectos del conocimiento, los pescadores habrían de diluirse lentamente en la elaboración teórica de la ciencia; aquel primer acercamiento acaecido en el mundo antiguo fue, para los enciclopedistas, superado con la aparición del hombre emancipado por la razón. La ciega creencia de que la humanidad iba vendada por la tradición hizo una generación de hombres fervorosos promotores de la ciencia nueva. Su fervor atravesó incluso océanos, contaminando a una generación imberbe de americanos en plena emancipación paternal.

\section{Los enciclopedistas europeos y la creación de una academia científica chilena}

La llegada de la independencia colonial impactó notablemente a los recién creados estados nacionales del cono sur americano. La victoria, sin embargo, desnudó la ignorancia del mundo criollo por su propio ser; los estados nacionales, inclusive antes de poder preguntarse quiénes eran sus ciudadanos, se vieron acosados por conocer sus territorios y sus potencialidades económicas. La naciente geopolítica de fronteras hizo imperioso el conocimiento de las riquezas inmediatas y futuras, además de los posibles conflictos con los estados vecinos (Barros Arana, 1898). Puntualmente en Chile, faltos del capital humano idóneo y con una universidad nacional en suspenso, el gobierno liberal del general Ramón Freire (1823-1826) hubo de iniciar actividades puntuales para captar a diversos intelectuales desde la Europa continental, en pos de realizar la doble tarea de construir un mapeo geográfico-botánico e histórico-identitario del país. El botánico francés Claude Gay (1800-1873) apareció como la entidad central en este nuevo desafío.

Invitado a viajar a Chile por un militar napoleónico (Pedro Chapuis), Gay accedió a asumir el desafío encomendado; sin embargo sus planes se vieron frustrados por la renuncia de Freire y la inestabilidad política del país - debido al conflicto entre liberales y conservadores -, obligando al botánico a emplearse en cargos menores de enseñanza (Barros Arana, 2005, p.97). El triunfo final de las facciones conservadoras - y la paz militar impuesta posterior a 1829 - permitió a Gay retomar sus planes originales al ser contratado (1830) por el presidente Ovalle para emprender la titánica tarea de realizar un recuento botánico, mineralógico, de fauna e histórico del país.

$\mathrm{Su}$ formación enciclopédica y las circunstancias políticas al momento de su llegada configuraron la visión de Gay en torno a la conducción y formas que debía asumir el país. Impresionado por las potencialidades de Chile, el naturalista reflexionó sobre las circunstancias que hacían imposible el correcto desarrollo de la púber nación: la sangre y el desorden. El profundo mestizaje de los sectores desposeídos y trabajadores incidía, según él, en la desidia popular y la falta de adopción de las formas modernas de trabajo. Propuso una forma de solucionar aquello, buscar la simiente de la nación chilena, su semilla impoluta: el mundo agrario. ${ }^{2}$ Dibujando, por tanto, en sus tomos relativos a la agricultura, un monumento al progreso, en palabras de Rafael Sagredo. La apertura económica significaba, para Gay, la 
posibilidad real del crecimiento del país junto con afirmar que en el campo descansaba el poder del Estado - bajo la tríada, orden, trabajo y familia -, desdeñando la capacidad de los sectores artesanales urbanos como entes de un desarrollo sustentable (Sagredo, 2009a, p.LII, LVIII-XLIX).

Relacionado al mundo pesquero, nuestro autor desarrolló una visión negativa. El botánico consideraba los pescadores indisciplinados y de corta inteligencia. A través de sus tomos de botánica y zoología, mencionó habitualmente el encuentro con "hábiles pescadores" que le entregaron informaciones o algún elemento para observar (Gay, 1848, p.142). Su habilidad, por tanto, radicaba en la acción de la pesca; reproducía Gay la división enciclopédica entre el hacer y el pensar; el pescador era un resumidero de informaciones y aquel era su valor, nunca sus percepciones de los fenómenos (Porter, 1922-1925, p.91; Sagredo, 2009b, p.XLV).

Contemporáneo a Gay, Charles Darwin (1809-1882) visitó el país (entre 1832 y 1835) también comenzó a consolidar la visión despectiva e infamatoria de la intelectualidad científica hacia el mundo pesquero. Absorto en un extenuante trabajo de campo, Darwin pudo recorrer costas y valles del país, en tanto anotaba las características más relevantes de la población autóctona (Darwin, 2005, p.158). Conocedor de la obra de Gay, compartió con él sus impresiones relativas a la situación social del país, refiriéndose con desdén para con el mundo pesquero, en tanto exaltaba al mundo campesino como un repositorio de la identidad nacional (Darwin, 1945, p.52).

Hacia la década de 1840, Ignacio Domeyko (1802-1889), profesor polaco de mineralogía, asentado en Coquimbo (región ubicada a 460 kilómetros al norte de la capital Santiago, en torno a los $29^{\circ}$ paralelo sur), emprendió un curioso viaje de exploración a la inhóspita Araucanía - distante seiscientos kilómetros al sur de la capital, encontrándose para el período aún indómita y controlada por fuerzas mapuche -; su extenso relato evidenciará una enjuta descripción del mundo pesquero, particularmente de la población de la ciudad costera de Penco (puerta norte de la Araucanía, asentada a 515 kilómetros al sur de Santiago) descrita como: "unas pocas familias de pescadores levantan allí sus chozas en medio de los escombros de los antiguos templos y cuarteles" (Domeyko, 2010, p.17). La aparentemente inocua frase debe leerse dentro del contexto del relato, como puede verse en el extenso comentario que ahora presentamos:

En efecto, en los tiempos que vivimos, pocas palabras hay que se repitan con más frecuencia, entre la gente ilustrada, que la palabra civilización, y pocas tal vez cuyo sentido sea menos claro y susceptible de interpretaciones más inciertas y vagas.

Si bajo este nombre comprendemos (lo que muchos civilizadores entienden) el trato exterior del hombre, su modo de vestirse, las comodidades que sabe proporcionarse, un cierto lujo y el uso de los útiles más necesarios para la vida doméstica, su habitación y el modo en que reside en ella; si, en fin, bajo este nombre se entiende la industria del hombre, es decir, cierta inteligencia que le sirve para mejorar su bienestar físico, su modo de pelear y de negociar con sus vecinos, una cierta perspicacia y casi malicia en sus relaciones con sus semejantes, confieso que, si esto solo se llama civilización, los indios araucanos no son salvajes, y tal vez son más civilizados que una gran parte de la plebe chilena y muchos de sus civilizadores de la frontera (Domeyko, 2010, p.61). 
Indefectiblemente la exaltación de los sectores indígenas, en este juego de oposiciones, veía su anverso en las poblaciones mestizas. El pescador, criollo o mestizo, era una entidad corrompida e incivilizada, comparado con el gallardo y orgulloso araucano o, inclusive, el campesino hacendado (Pinto Rodríguez, 2010, p.XXI). Los pilares fundadores de la ciencia en Chile, tanto vía sus enseñanzas como escritos, dividieron notablemente la población nacional en grados y niveles; políticamente los pescadores, y sus formas de vida trashumantes, eran prescindibles al momento de la construcción de la novel república.

\section{La cotidianeidad de la pesca y la disputa por sus saberes: el pescador visto por la academia ictiológica chilena}

Instalados en puestos de docencia y dirección gubernamental, los primeros científicos contratados por los gobiernos republicanos comenzaron a construir una academia científica nacional. La profundización de los gobiernos conservadores y la particular acción del varias veces ministro Antonio Varas impulsarán la llegada de constantes oleadas de inmigrantes del centro norte europeo, resaltando en el ámbito científico los hermanos Bernardo y Rodulfo Philippi (Barros Arana, 1904). Los extranjeros, nucleados por la Universidad de Chile (nacida de las cenizas de la colonial Universidad de San Felipe), comenzaron a educar a una nueva generación de profesionales que se alejaron de la tradicional matriz formativa de las élites tardo-coloniales (abogados, teólogos y clérigos) (Saldivia, 2003). La profesionalización de la ciencia fue acompañada por un ordenamiento del conocimiento y su lenguaje, el francés era el lenguaje común y el método positivo su piedra filosofal.

Con propiedad, la naciente academia científica de Chile llamó a sus coterráneos a involucrarse con ellos en el impulso de la ciencia. Amparados en notas aparecidas en publicaciones periódicas (particularmente El Mercurio de Valparaíso) ${ }^{3}$ y en los Anales de la Universidad de Chile, intentaron proponer un cambio de visión al mundo político nacional; la falta de iniciativas serias de apoyo a la investigación científica en Chile mostró las diametrales diferencias entre el joven mundo académico y su vetusto padre político (Saldivia, 2005, p.41-42, 46, 106). Únicamente durante la segunda mitad del siglo diecinueve, las juventudes oligárquicas fueron seducidas por las ciencias; los conflictos asociados a la separación de la Iglesia y el estado polarizaron a la juventud e impulsaron a un notable porcentaje de jóvenes al estudio de las ciencias, revelándose, ahora, el positivismo y la ciencia como la materia más revolucionaria impartida en las aulas chilenas. ${ }^{4}$

Sin desconocer lo anterior, aún las huestes científicas eran exiguas y, particularmente al caso que nos atañe, casi nulas las menciones al mundo pesquero artesanal y sus trabajadores. Realizado un somero recuento de los trabajos publicados en los Anales de la Universidad de Chile desde 1843 a 1900, podemos notar que no existieron tesis relativas a problemáticas pesqueras. ${ }^{5}$ La ictiología era una ciencia desconocida dentro de las aulas nacionales, los naturalistas se habían abocado a la recopilación de especímenes, mas no a su estudio acabado. Frente a la agronomía y minería, motores y sustentos de la economía y dieta nacional, la costa y sus productos no suscitaron la misma preocupación del mundo científico y político. ${ }^{6}$

La concreción de las primeras sociedades científicas nacionales, durante la última década del siglo, vio recompensados los esfuerzos de los maestros extranjeros. Refugiada en su seno, 
una nueva generación de jóvenes se abocó a la creación de un nuevo lenguaje social. ${ }^{7}$ Los noveles científicos intentaban trasladar las metodologías científicas al país, adaptando sus formas al ambiente y plasmando en sus alegorías las caras de los connacionales. Imbuidos en el espíritu de su tiempo, despreciaban las formas tradicionales, vivían cegados por el progreso y las formas que el mismo proyectaba: industria, ciencia y felicidad, las tres hermanas que caminaban juntas entre los siglos (Bury, 1971; Norambuena, 2003).

Transitando en un camino en paralelo, la Armada de Chile comenzó a interesarse en el desarrollo científico de sus oficiales. La resaca de la eufórica celebración del triunfo sobre Perú y Bolivia en la guerra del salitre (1879-1884) arrojó un dejo de duda relativo a la capacidad de Chile de asumir su nuevo rol militar en el Pacífico. Prontamente se percibió que la debilidad nacional estaba radicada en su precaria base informática, acelerando la construcción de matrices geográficas, geodésicas y cartográficas de las costas, junto con incentivar el conocimiento de la vida marina que ahí habitaba. La primera acción en concreto fue la fundación del Club Naval, con asiento en el puerto de Valparaíso desde 1885, que tuvo tres objetivos: propender a la especialización científica de los oficiales, crear una publicación (Revista de Marina) donde difundieran sus resultados y establecer una biblioteca (Fierro, 2005, p.182). La concreción de las tres iniciativas, el mismo año de su fundación, hizo del Club el semillero de una generación científica en la Armada, permitiendo que, además, sus oficiales se relacionaran con el mundo científico nacional (Saldivia, 2005, p.125-126).

Construida una base científica nacional, fusión de la oficialidad con el mundo civil, el espacio chileno comenzó a ser inspeccionado, diseccionado e investigado. Hambrientos, los científicos nacionales se abocaron a trabajar en la pesca y en los pescadores. Importante esfuerzo que los alejó de sus matrices enciclopédicas y los territorializó en la realidad material del país. La riqueza del salitre y la mayor concentración urbana hizo imperioso al Estado la necesidad de contar con mayores y más constantes flujos de alimentos, imaginándose a los sujetos involucrados en las áreas alimentarias como actores en posiciones estratégicas. Posiciones que no podían ser abandonadas a la especulación o la desidia.

\section{El espejo distorsionado}

Entregados a la educación positiva, los científicos y militares habían desarrollado durante el siglo XIX un extenso recorrido por sus metodologías e implicancias políticas, adquiriendo una confianza extrema en la pertinencia de su método y objetivos. Sin embargo, la teoría era el continente del conocimiento, no su interior; el 'saber' aún residía en los sujetos no científicos. Paradoja propia de las sociedades que importaban el conocimiento, siendo así, la primera tarea del mundo científico finisecular correspondió a la extracción del conocimiento desde quién lo detentaba (el pescador) y su apropiación para encausarlo al correcto uso: el científico.

Singular en este diálogo habría de ser el médico de la Armada, Federico Delfín (1851-1904), unánimemente presentado como el primer ictiólogo chileno. Él representó la expresión acabada del maridaje entre la formación científica de corte europeo y el conocimiento de los espacios y sociedades litorales del país (Porter, 1909, p.23; 1914-1916, p.134; Godoy, 1988, p.50-51). Delfín vio como una necesidad apropiarse de las informaciones que, a sus ojos, se perdían en las mentes y acciones de los trabajadores del mar. ${ }^{8}$ Consideraba, debido a su formación, que el conocimiento de los pescadores era ilusorio, sólo manejaban informaciones desperdigadas 
y reunidas sin sentido, siendo simples repositorios de impresiones pre científicas. ${ }^{9}$ El párrafo más notable, en esta línea, se extrae de la presentación que realizó en el séptimo congreso científico chileno de 1903. La exigua censura en sus palabras y el desprecio evidente por el otro, que trasunta la exposición, nos lleva a proponer que el ambiente académico cerrado y protector habría inhibido el pudor del autor al momento de expresar cabalmente su juicio en torno al pescador (herramienta a esta altura) y su disputa por hacer valer su conocimiento equiparable y contrastante con el científico académico:

Una pesca de esta naturaleza (artesanal) es falta de base, es fatal y sin embargo, hay quienes tienen algunos datos que emplean con cierta astucia; algunos poseen verdaderos conocimientos que una larga práctica, unida a la observación, les ha proporcionado. Pero esto es solo en cierto orden. Ya en lo que se refiere a la preparación de algunos útiles, ya en sus manejos en lo que son eximios y a veces prácticos de los lugares donde pueden obtener los mayores beneficios. Hay algunos que dicen poseer secretos legados de sus mayores, que solo trasmiten a sus descendientes; pero esto se refiere solo a lugares de pesca, conocimiento empírico, o más bien dicho, imaginario, y los más, un poco de aquí y otro de allá, ninguno tiene idea cabal de su oficio, como que solo son operarios prácticos y en su mayor parte sin instrucción ni espíritu de observación para sacar todo el partido a que se presta el estudio atento a las leyes naturales en el régimen de vida de los peces (Delfín, 1903, p.303-331).

Delfín expone claramente la necesidad de hacer producir la pesca en beneficio del país. Como funcionario de las fuerzas armadas comprendía los cambios sucedidos en los estados antes de la primera guerra mundial. La acelerada modernización de los estados del Cono Sur americano estuvo asociado a una expansión burocrática de su base - generación de mayores empleos y creación de una clase media comprometida con el Estado y sus representantes - y tecnocratización de su cúspide - tecnificación de los grupos de apoyo de los gabinetes ministeriales (Silva, 2006, p.182-183). Aquello implicaba que los científicos pasaban a ser parte de los gabinetes ministeriales, sus consejos técnicos fueron primordiales al momento de proyectar países de acelerado crecimiento en un mundo en tensión y conflicto por la extracción de materias primas. Inmerso en tal coyuntura, el científico apareció como la superación natural del pescador, rescatando al mar chileno de su cautiverio por la ignorancia. ${ }^{10}$

Profundamente comprometidos con su nuevo rol dentro del Estado - en gran medida por sentirse reconocida su labor, por primera vez -, los científicos superaron sus primeros comentarios orientados a denostar el conocimiento del otro, tornándose a exponer las primitivas e improductivas formas de extracción del mundo artesanal (Pomar, 1900, p.4). De los diversos escritos emanados en su momento, podemos extraer tres afirmaciones centrales que definen la visión del mundo artesanal desde el prisma científico. La primera afirmación fue quizás la más tajante, insinuaron el cariz a-patriótico de los pescadores artesanales, ya que en su constante especulación de los precios impedían acceder de manera económica a los productos marinos (Pomar, 1900, p.20; Delfín, 1903, p.309-310; Reed, 1938, p.302); la segunda afirmación aseveró que los burdos rudimentos de pesca utilizados devastaban los ambientes marinos, particularmente por el uso de dinamita que destruía el fondo oceánico y los peces y moluscos en maduración (Pomar, 1900). Y la tercera afirmación residió en la idea de que el pescador artesanal no consumía de forma inteligente sino depredaba. Los intereses 
industriales que lentamente fueron integrándose en las sociedades científicas se dedicaron, particularmente, en instalar este supuesto. La señora Laurencia Collongues Baragnes, esposa del conde Emile de Solminihac (ambos con intereses en las conserveras de la provincia de Llanquihue (meridional región ubicada entre los grados 40 a 43 del paralelo sur), afirmó que el pescador artesanal era un 'enemigo' de los peces y mariscos. Su deseo por la obtención de una ganancia elevada y rápida lo hacía presa de especuladores, cuando no lo era él mismo; presentando al pescador como un ente contrario a la conservación y reproducción de su propia fuente de trabajo. En sus palabras:

el agotamiento que la lógica hacía prever, pero nada importaba! Mientras existían ostras, nadie se preocupaba! Por el contrario, se pagaba la prima al que pescaba más, y como pescan sin tino, se comprende el resultado! ... El precio subido que se ha pagado en estos últimos tiempos por ese molusco (ostra) ha inducido al pescador a recoger sin piedad todo lo que encuentra limpiando totalmente las playas (Solminihac, 1929, p.94-95, 97).

La diferencia de objetivos llegó a niveles de imposibilidad de diálogo. Inclusive Delfín llegó a recomendar a los investigadores el buscar sus especímenes entre los desechados o irrelevantes para el pescador artesanal. El simple desprecio del último podría ser prenda de valor asegurada para el investigador. ${ }^{11}$ Hasta los roles ya no eran los mismos. Los científicos durante el siglo diecinueve dependieron del apoyo de niños y hombres sin educación para abastecerse de muestras biológicas, botánicas y minerales o para obtener informaciones específicas. Sin embargo, durante las primeras décadas del siglo veinte, el discurso científico transformó aquella dependencia en un factor positivo para los individuos que entregaban la información. El mundo científico equiparaba la ecuación, cambiando información por educación, inclusive sin que los sujetos lo supieran. Carlos Oliver Schneider, encargado del museo de la ciudad de Concepción y llamado el precursor de la biología marina en el país (Yáñez, 1950), rememorando sus propios actuares entrega luces sobre la situación antes señalada:

Y de mi contacto personal con los niños, en los diez años que llevo en la jornada, que día a día llegan al Museo trayendo cualquier cosa curiosa, una pirita o un ave, o muchas veces un dato valioso, también se me ocurre que esa modesta labor de cooperación que realiza diariamente el Museo, puede traer para el futuro, mayores bienes y realidades para la ciudad. Realiza, quizás con ello, nuestro Museo, una obra social no sospechada hasta ahora (Oliver, 1929, p.18-19).

En consonancia con las tendencias norte atlánticas, la consolidación de la academia científica fue aparejada de la división en grupos temáticos individuales, la misma identidad del naturalista fue reemplazada por la del especialista. Específicamente en la ictiología, en 1909, Bernardino Quijada Burr fundó la Estación Marina del Pacífico Sur y el Museo Oceanográfico de la ciudad-puerto de San Antonio (límite costero del Chile central). Empero su corta duración, significó el primer esfuerzo de profesionalizar los impulsos científicos dispersos a lo largo del siglo diecinueve (Bahamonde, 2005, p.47-48). Anteriormente nunca la ciencia había estado tan desagregada del saber tradicional; había acabado el proceso de extracción de conocimiento y llegaba, en tanto, el momento de concentrarse a extraer sus propias conclusiones. Desaparecía nuevamente el sujeto de la acción (pescador), el acto de 
pescar se pensaba ya racionalizado por las empresas pesqueras. El pez había dejado de ser un misterio. Fuera del mundo iluminado por la ciencia, la pesca artesanal vivía sus propias temporalidades y abastecía silenciosamente a los nutridos mercados urbanos del país. Sin embargo, para la intelectualidad, su existencia aparecía como un vestigio arqueológico de un hacer ya caduco, siendo referidos sus gestores y formas de manera pintoresca y folclórica. ${ }^{12}$

\section{Consideraciones finales}

La revolución neo cartesiana y, posteriormente, positiva influyó directamente en la creación del primer conocimiento científico sistematizado en Chile; el arribo de profesores europeos nutrió a varias generaciones de investigadores que consolidaron su vida a llevar el progreso al país. Deslocalizados, los profesores europeos se abocaron a presentar sus conocimientos como una teoría universal - posible de intercambiar y trasladar a los territorios sin pérdida de su validez intrínseca. Aprehendidas las bases teóricas del saber nuevo, los estudiantes nacionales se volcaron al estudio del país, sin embargo, observaron su ignorancia en comparación con los actores tradicionales; obligado a pedir o comprar informaciones, el científico comenzó a desarrollar la certeza de la imposibilidad del diálogo: siendo erradas las bases del conocimiento popular, el científico debía mantenerse higiénicamente cercado para poder realizar su labor. ${ }^{13}$

El desdén hacia la religión popular y los sectores tradicionales, que caracterizó a la generación enciclopédica y positiva, fue reproducido en Chile durante los conflictos de separación del estado de la Iglesia romana (Serrano, 2008). Los jóvenes estudiaban ciencia por convicciones políticas, posterior era el interés particular por la especialidad. Aquel compromiso político con el futuro del país entraba en conflicto (aparente) con formas de conductas tradicional que, no obstante adherir al modelo nacional, distaban de considerar que la mejor consecución económica del país era la eliminación de sus faenas productivas de corte artesanal. Tal petición autoflagelante se apoya no en la búsqueda de una mejora conjunta para el país sino, más bien, en el carácter de prescindible que instaló el medio científico en relación al conocimiento del otro.

Cuando se firmó la declaración sobre la ciencia y el saber científico en 1999 - punto inicial de este trabajo - se propuso que la ciencia institucionalizada respetaría a las otras "creatividades humanas"; no obstante obviaba tal declaración que las informaciones de base de la ciencia moderna fueron extraídas desde las "creatividades humanas" (de corte científico no occidental). No mencionar lo anterior induce a una dicotomización irreal del saber humano, perpetuando una tolerancia de exclusiones, que reconoce nichos y campos privados en desmedro de la integración horizontal de los saberes.

La propuesta de nuestro trabajo ha sido la exposición en un período limitado (1829-1909), mostrando cómo la ciencia ictiológica en Chile se nutrió de las vertientes norte atlánticas e inició una conflictiva relación con las poblaciones pesqueras en su deseo por hacerse de su conocimiento y codificarlo según la matriz científico-moderna. Imposible de saber hoy si los pescadores asimilaron o integraron informaciones dadas por los científicos a fines del siglo XIX (aquello solamente valdría una investigación); contraria situación ocurrió con los científicos, ellos sí integraron el conocimiento extraído de los pescadores. A través del análisis de tales informaciones, los científicos comenzaron a asumir un protagonismo inusitado - 
inclusive mayor que las informaciones -, situación que los impulsó a participar directamente en la toma de decisiones gubernamentales. El siglo XX mostró que las decisiones tomadas se orientaron a desapoderar a los sectores de pescadores tradicionales, sustento prístino de su ciencia. Habrán de transitar numerosos lustros antes que dentro de las reuniones científicas retornen las reivindicaciones del saber tradicional. La pregunta es si habremos de esperar muchos más para poder ver los resultados de tal relación de respeto y trabajo en conjunto.

\section{NOTAS}

* Este artículo se enmarca dentro del proyecto del Fondo de Fomento al Desarrollo Científico y Tecnológico (Fondef), n.D08I1107, “Chile Litoral 2025: modelo de gestión territorial para asentamientos de pescadores artesanales", realizado entre los años 2010-2014.

${ }^{1}$ Aquella visión pudo ser transmitida del trabajo del inglés Ephraim Chambers, creador de la Cyclopcedia: or, an universal dictionary of arts and sciences, en 1728 (antecedente directo de la Encyclopédie de Diderot). En su primer tomo se aborda la pesca (páginas 42 a 48), sin embargo, aunque explica las temporalidades, sus herramientas y niveles de productividad, aparece como una faena sin operarios. Inclusive la palabra pescador (fisherman) no está incluida en el diccionario. Véase Chambers (1728).

${ }^{2}$ Un párrafo ilustrativo de lo anteriormente planteado, entre otros, es el siguiente: “Así, pues, (la agricultura) ha permanecido por espacio de siglos en un estado completamente sedentario, supeditada además por el elemento araucano, único que podía proporcionarle trabajadores y que debía necesariamente inocular en el trabajo es (sic) espíritu de incuria y de rutina innato en el carácter indio y cuyas consecuencias no podían menos que contrariar las reformas y contener todo progreso" (Gay, 2009, p.3).

${ }^{3}$ Fundado en 1826, en el mayor puerto de Chile, Valparaíso, representó desde sus inicios la voz de los sectores económicos pujantes y progresistas del país, radicando ahí su interés especial en la difusión de las ciencias en el país. Para un estudio histórico y documental de sus aportes durante el siglo diecinueve chileno, véase Cid (2009).

${ }^{4}$ El fervor juvenil de sus adherentes puede ser recreado en el relato íntimo que realizó Jorge Lagarrigue, uno de los principales impulsores del positivismo chileno, en 1875: "Hago este trabajo porque creo un deber de mi parte propagar esa filosofía que ha alumbrado mi espíritu y porque creo que el positivismo es el punto final hacia el cual marchan las sociedades en sus constantes progresos. Las ciencias, llegadas casi todas a un estado realmente positivo, preparan el camino. ... yo quiero servir a mi país, combatiendo el error, esparciendo y defendiendo la verdad. Creo hacer esto, propagando las grandes doctrinas de la filosofía positiva" (Lagarrigue, 1980, p.144).

${ }^{5}$ Durante los últimos años, la Universidad de Chile ha colocado en línea gran parte de su colección de Anales (primera serie), los cuales pueden ser consultados en: http://www.revistas.uchile.cl/index.php/ ANUC/issue/archive.

${ }^{6}$ Aquí, el padrón cultural de consumo influyó, la pobreza de la población y la preferencia por las legumbres y las carnes rojas hizo de Chile un país con reducido consumo de productos extraídos del mar. En consonancia a lo expuesto, las miras científicas y políticas no se orientaban a las capacidades productivas de las costas (Couyoumdjian, 2009, p.57-58).

${ }^{7}$ Resonancia adquiere aquí la afirmación de Thomas Kuhn (1998) relativa a que las revoluciones tecnológicas van asociadas a un cambio en el lenguaje, alterándose los criterios con que tales conceptos se relacionan entre sí y con el mundo observado. Los cambios en las metáforas científicas, parafraseando a Kuhn (p.8889), entregan la flexibilidad necesaria al científico para poder instalar una información que aparece como novedosa dentro de un modelo mental ya establecido.

${ }^{8}$ Delfín escribió que para extraer la información de los pescadores había "gastado dádivas y procurando tino he podido sacarles algunos datos". Incluso llegó a exponer que en aquello residía el mayor valor de su investigación: "No creo pueda tener cabida en este trabajo todos los datos que poseo en mis notas llevadas pacienzudamente i estudiosamente escrutados como verdaderos en el trascurso de más de quince años, en distintas épocas del año i en todo el litoral de nuestras costas, desde Camarones hasta Punta Arenas, donde he encontrado pescadores a quienes interrogar". Para la primera cita, véase Delfín (1900b, p.420); para la segunda, Delfín (1898, p.93). 
${ }^{9}$ Comentando así cómo sabían los boteros si la pesca sería favorable: "presintiendo una pesca favorable y otros adversa, según apreciaciones deducidas, las más, del estado del tiempo, de las mares y de la luna" (Delfín, 1900b, p.424-425).

${ }^{10}$ La guerra del salitre hubo de colocar a Chile en una encrucijada. Su aparición como posible potencia militar en el Pacífico hizo que sectores de gobierno intentaran impulsar una industria derivada de la riqueza redituada por el conflicto. Sin embargo, el apresuramiento sólo mostró las precariedades científicas e industriales del país. El presidente de la Sociedad Científica de Chile fue tajante al afirmar que: "Porque los pueblos, como los individuos, que crecen i prosperan con desusada rapidez, retrogradan y mueven con velocidad proporcional. Pues bien, mientras Chile no logre andar un buen espacio de trecho que lo separa del progreso europeo, no hallará conveniencia ni tendrá los medios de cultivar la ciencia por la ciencia" (Pérez, 1900, p.XVI).

11 "Es de saber que los pescadores tienen su manera de clasificar la pesca, en pescados de 'venta valiosa, fácil i nula', correspondiendo a estos últimos los peces que nadie compra por 'desconocidos', por no saber si es comestible, buena o mala su carne, de lo que los aficionados pueden aprovechar i encontrar los ejemplares más interesantes abandonados en la orilla de la playa o al muy bajo precio de mano de los pescadores si es que no manifiestan mucho interés" (Delfín, 1900a, p.150).

${ }^{12}$ En ámbitos pictóricos, la obra de Arturo Pacheco Altamirano (particularmente sus obras sobre Angelmó y los astilleros de Valdivia) rescató aquella visión colorida del mundo pesquero artesanal, insertándole un dejo de nostalgia cual organismo en proceso de muerte. Igual imagen, ahora desde la literatura, se puede extraer de la obra de Manuel Rojas en su Hijo de ladrón, ambientada en un Valparaíso enmohecido y apagado (Nazare, 1964; Rojas, 1951).

${ }^{13}$ Edwyn Reed (1938, p.301) nos transcribe una inusual conversación con pescadores del puerto de Valparaíso, ansioso por conocer las impresiones de la población en torno a la implementación de un nuevo puerto en la ciudad - que debería bajar los precios al aumentar la competencia -, "he conversado con muchos pescadores. Se ríen de mi pregunta. Varios afirman que al contrario, deben subir los precios porque gastarán más ellos (aunque no veo cómo van a gastar más). Tampoco aumentará la pesca, dicen, porque pescarán igualmente, pero estarán más seguros y cuidarán más sus botes!!!".

\section{REFERENCIAS}

BAHAMONDE NAVARRO, Nibaldo. Homenaje al profesor Parmenio Antonio Yáñez Andrade (1902-1977). In: Figueroa, Eugenio (Ed.). Biodiversidad marina: valoración, usos y perspectivas ¿hacia dónde va Chile? Santiago de Chile: Universitaria. p.45-55. 2005.

BARROS ARANA, Diego.

Historia general de Chile, t.15. Santiago de Chile: Universitaria. 2005.

BARROS ARANA, Diego.

El doctor Rodulfo Amando Philippi: su vida i sus obras. Santiago de Chile: Cervantes. 1904.

BARROS ARANA, Diego.

La cuestión de límites entre Chile i la república

Arjentina. Santiago de Chile: Roma. 1898.

BRAUDEL, Fernand.

Civilisation matérielle, économie et capitalisme: $\mathrm{XV}^{\mathrm{e}}$ XVIII ${ }^{e}$ siècle. t.1, Les estructures du quotidien: le posible et l'impossible. Paris: Armand Colin. 1979.

BURY, John.

La idea del progreso. Madrid: Alianza. 1971.

CHAMBERS, Ephraim.

Cyclopædia: or an universal dictionary of arts and sciences. Disponible en: http://digital.library. wisc.edu/1711.dl/HistSciTech.Cyclopaedia01. Consultado el: 14 set. 2011. 1728.

CID, Gabriel (Compilador).

El Mercurio chileno. Santiago de Chile: Dibam. 2009.

COUYOUMDJIAN, Juan Ricardo.

El mar y el paladar: el consumo de pescados y mariscos en Chile desde la independencia hasta 1930. Historia, Santiago de Chile, v.41, n.1, p.57-107. 2009.

DARWIN, Charles.

Darwin en Chile (1832-1835): viaje de un naturalista alrededor del mundo. Santiago de Chile: Universitaria. 2005.

DARWIN, Charles.

Viaje de un naturalista alrededor del mundo. Buenos Aires: El Ateneo. 1945.

DELFÍN, Federico.

Los congrios de Chile. In: Congreso Científico

Jeneral Chileno, 7., 1903, Valdivia. Actas...

Santiago de Chile: Imprenta Cervantes. p.303-363. 1903. 
DELFÍN, Federico.

Algunas observaciones sobre la colecta, conservación i remisión de peces para los museos. Revista Chilena de Historia Natural, Valparaíso, v.4, n.9. 1900a.

DELFÍN, Federico.

Voracidad de la Bdellostoma polytrema de Girard. Actes de la Sociétè Scientifique du Chili, Santiago de Chile, v.10, p.419-427. 1900b.

DELFíN, Federico.

Catálogo de los peces de Chile. Revista Chilena de Historia Natural, Valparaíso, v.7, n.2. 1898.

DÍAZ DE LA SERNA, Ignacio.

El artículo 'América' en la Enciclopedia de Diderot y D'Alembert (primera parte). Norteamérica, Ciudad de Mexico, v.4, n.1, p.163-204. 2009.

DOMEYKO, Ignacio.

La Araucanía y sus habitantes. Santiago de Chile: Pontificia Universidad Católica de Chile; Dibam; Cámara Chilena de la Construcción. 2010.

FIERRO, Juan.

Recuerdos del Club Naval de Valparaíso (narrados por su edificio). Revista de Marina, Valparaíso, v.122, n.885, p.182-183. 2005.

GAY, Claudio.

Prólogo. In: Gay, Claudio. Historia física y política de Chile: agricultura. t.1. Santiago de Chile: Pontificia Universidad Católica de Chile; Dibam; Cámara Chilena de la Construcción. p.3-31. 2009.

GAY, Claudio.

Historia física y política de Chile: zoología. t.2. París: Maulde et Renou. 1848.

GODOY, Hernán.

Desarrollo histórico del sector pesquero en Chile. Ambiente y desarrollo, Bogotá, v.4, n.1-2, p.45-56. 1988.

GÓMEZ SALAZAR, Mónica; VILLAR, Mauricio del.

El concepto de propiedad y los conocimientos tradicionales indígenas. En-claves del pensamiento, Ciudad de Mexico, v.3, n.5, p.115-135. 2009.

KUHN, Thomas.

¿Qué son las revoluciones científicas? In: Kuhn, Thomas. ¿Qué son las revoluciones científicas? y otros ensayos. Barcelona: Altaya. p.55-93. 1998.

LAGARRIGUE, Jorge.

Trozos del diario íntimo. In: Zea, Leopoldo. Pensamiento positivista americano. t.1. Caracas: Ayacucho. p.143-161. (Selección y prólogo de Leopoldo Zea). 1980.

NAZARE, Jacobo.

Arturo Pacheco Altamirano: vida y obra.
Concepción: Editorial Universidad de Concepción. 1964.

NORAMBUENA, Carmen.

Imagen de América Latina en la exposición universal de París de 1889. Dimensión Histórica de Chile, Santiago de Chile, n.17-18, p.87-122. 2003.

OLIVER SCHNEIDER, Carlos.

El museo de Concepción, lo que es y lo que ha hecho. Actes de la Sociétè Scientifique du Chili, Santiago de Chile, v.36. 1929.

PÉREZ, Clodomiro.

Discurso del presidente Clodomiro Pérez Canto, sesión general del 19 de mayo 1900. Actes de la Sociétè Scientifique du Chili, Santiago de Chile, v.10. 1900.

PINTO RODRÍGUEZ, Jorge.

Ignacio Domeyko: viaje a la Araucanía en el año 1845 y otros documentos sobre la frontera. In: Domeyko, Ignacio. La Araucanía y sus habitantes. Santiago de Chile: Pontificia Universidad Católica de Chile; Dibam; Cámara Chilena de la Construcción, p.IX-LVIII. 2010.

POMAR, Luis.

Memoria sobre la pesca en Chile. Valparaíso: Talleres tipográficos de la Armada. 1900.

PORTER, Carlos.

La contribución al estudio de las ciencias naturales de Chile por los sabios franceses. Actes de la Sociétè Scientifique du Chili, Santiago de Chile, v.32-35. 1922-1925.

PORTER, Carlos.

Discurso de Carlos Porter sobre la contribución de la Socièté Scientifique du Chili a los estudios histórico-naturales, Santiago de Chile, 28 de diciembre de 1916. Actes de la Sociétè Scientifique $d u$ Chili, Santiago de Chile, v.24. 1914-1916.

PORTER, Carlos.

El doctor Federico Teobaldo Delfín: fallecido en 22 de junio 1904. Actes de la Sociétè Scientifique du Chili, Santiago de Chile, v.19. 1909.

REED, Edwynd.

El pescado en la alimentación de los chilenos. In: Congreso Científico General Chileno, 9., Valparaíso. Santiago de Chile: Abel Palma. t.1. p.299-303. 1938.

ROJAS, Manuel.

Hijo de ladrón. Santiago de Chile: Nascimento. 1951.

SAGREDO, Rafael.

La 'agricultura' de Claudio Gay. Un panorama social de Chile en el siglo XIX. In: Gay, Claudio. Historia física y política de Chile: agricultura. t.1. Santiago de Chile: Pontificia Universidad Católica de Chile; Dibam; Cámara Chilena de la Construcción. p.IX-LXXI. 2009a. 
SAGREDO, Rafael.

Los documentos de la 'historia' de Gay: la confirmación de su método histórico. In: Gay, Claudio. Historia física y política de Chile: documentos. t.1. Santiago de Chile: Pontificia Universidad Católica de Chile; Dibam; Cámara Chilena de la Construcción. p.IX-LXIV. 2009b.

SALDIVIA MALDONADO, Zenobio. La ciencia en el Chile decimonónico. Santiago de Chile: Ediciones Universidad Tecnológica Metropolitana. 2005.

SALDIVIA MALDONADO, Zenobio. La visión de la naturaleza en tres científicos del siglo XIX en Chile: Gay, Domeyko y Philippi. Santiago de Chile: Editorial Universidad de Santiago de Chile. 2003.

SALLES, Cecilia de.

A Encyclopédie de Diderot: de tratado a álbum ilustrado: observações sobre os riscos de interpretações editoriais. Anais do Museu Paulista: História e Cultura Material, São Paulo, v.1, n.1, p.293-296. 1993.

SERRANO, Sol.

¿Qué hacer con Dios en la República? Política y secularización en Chile (1845-1885). Santiago de Chile: Fondo de Cultura Económica. 2008.
SILVA, Patricio.

Los tecnócratas y la política en Chile: pasado y presente. Revista de Ciencia Política, Santiago de Chile, v.26, n.2, p.175-190. 2006.

SOLMINIHAC, Laurencia de.

La ostra y la ostricultura chilena. Actes de la Sociétè Scientifique du Chili, Santiago de Chile, v.36. 1929.

TINNALUCK, Yuwanuch.

Ciencia moderna y conocimiento nativo: un proceso de colaboración que abre nuevas perspectivas para la PCST. Quark, Barcelona, n.32, p.24-29. 2004.

\section{UNESCO/ICSU.}

United Nations Educational, Scientific and Cultural Organization/International Council for Science. Declaración sobre la ciencia y el uso del saber científico. Conferencia mundial sobre la ciencia para el siglo XXI: un nuevo compromiso. Budapest, 26 jun. a 1o jul. 1999. Disponible en: http://www.unesco.org/science/wcs/esp/ declaracion_s.htm. Consultado el: 14 sep. 2011. 1999.

YÁÑEZ, Parmenio.

El profesor Carlos Oliver Schneider, un precursor de la biología marina en Chile. Revista de Biología Marina y Oceanografía, Valparaíso, v.2, n.2-3, p.97-116. 1950. 